\title{
DISTRIBUCIÓN DE LA PATOLOGÍA QUIRÚRGICA DEL CUERPO UTERINO EN MUJERES CHILENAS SOMETIDAS A HISTERECTOMÍA EN UN HOSPITAL CLÍNICO UNIVERSITARIO
}

\author{
Paulina Merino O. ${ }^{2}$, Adriana Doren V. a , Nicolás Sáez O. ${ }^{3}$, Pilar Valenzuela M. ${ }^{3}$, \\ Alejandra Etchegaray B. ${ }^{4}$, Roger Gejman E. ${ }^{5}$, Mauricio Cuello F. ${ }^{1}$ \\ 1 Departamento de Obstetricia y Ginecología, Escuela de Medicina, Pontificia Universidad Católica de Chile. ${ }^{2}$ Servicio \\ de Ginecología y Obstetricia, Pontificia Universidad Católica de Chile. 3 Programa de especialización en Obstetricia y \\ Ginecología, Escuela de Medicina, Pontificia Universidad Católica de Chile. 4 Gineco-Obstetra, Universidad de Chile \\ (Hospital San Borja Arriarán). 5 Departamento de Anatomía Patológica, Escuela de Medicina, Pontificia Universidad \\ Católica de Chile.
}

a Interna, Escuela de Medicina, Pontificia Universidad Católica de Chile.

\section{RESUMEN}

Antecedentes: La histerectomía es la cirugía ginecológica mundialmente más frecuente. Pocas series analizan la distribución de los hallazgos patológicos en histerectomizadas. Objetivos: Describir la distribución de la patología uterina originada en el endometrio, miometrio y estroma, en histerectomías efectuadas en un centro universitario. Determinar la frecuencia en que coexisten patologías, particularmente cáncer incidental en histerectomías por condiciones presuntamente benignas. Métodos: Estudio retrospectivo de todas las histerectomías efectuadas entre los años 1991 y 2005. Análisis descriptivo de la distribución de las enfermedades benignas y malignas originadas en el cuerpo uterino. Resultados: Se realizaron 5683 histerectomías. En 4275 úteros se diagnosticaron lesiones de origen no epitelial, principalmente leiomiomas uterinos y adenomiosis. En las 2070 piezas con lesiones epiteliales (endometrio) los hallazgos más prevalentes fueron atrofia, pólipo endometrial e hiperplasia glandular del endometrio. Hubo coexistencia de patología miometrial y endometrial en 905 piezas quirúrgicas. En 240 casos no hubo lesiones en la biopsia $(4,2 \%)$. En el $1 \%$ de las histerectomías se encontró como hallazgo un cáncer ginecológico, siendo los dos diagnósticos más frecuentes asociados con esta situación, la metrorragia disfuncional perimenopáusica y el pólipo endometrial. Conclusiones: La distribución de los diagnósticos de la patología uterina es similar a la descrita por series internacionales. El hallazgo más común es el leiomioma uterino, frecuentemente asociado con adenomiosis. Se destaca el hallazgo incidental de cáncer de endometrio en histerectomizadas por metrorragia y/o pólipo endometrial; esto nos hace recomendar el uso rutinario del estudio biópsico preoperatorio en pacientes con metrorragia y de la biopsia contemporánea en casos de pólipo endometrial.

\section{PALABRAS CLAVE: Histerectomía, patología uterina, leiomioma, pólipo endometrial, cáncer incidental}

\section{SUMMARY}

Background: Hysterectomy is the most frequently performed gynecologic procedure worldwide. Few studies have been done to analysis the distribution of pathological findings in hysterectomies. Objectives: To determine the distribution of epithelial and non-epithelial pathology in patients undergoing hysterectomy for 
uterine disease in an academic centre. To establish what is the percentage and the etiology of concurrent pathologies and the incidence of unexpected cancer within patients undergoing surgery for a presumably benign condition. Methods: A retrospective study was done of all the patients undergoing hysterectomy between 1991 and 2005. In addition a descriptive analysis of the distribution of benign and malignant conditions originated in the uterine corpus was done highlighting the occurrence of incidental cancer among different preoperative diagnosis. Results: During the period, 5683 hysterectomies were performed. In 4275 cases a non-epithelial pathology was found, mainly, fibroids and adenomyosis. In 2070 cases endometrial pathology was diagnosed: atrophy, polyps and hyperplasia as the most prevalent findings. In 905 cases myometrial and endometrial pathology of uterine corpus coexists. In 240 cases any pathology was found (4.2\%). In $1 \%$ of hysterectomies an incidental gynecological cancer was found, and the two conditions more frequently associated were metrorrhagia and polyps. Conclusions: The distribution of etiologies for the uterine pathology, is similar to other international series. The most common finding is fibroid frequently associated to adenomyosis. It is important to highlight the incidental finding of endometrial cancer among patients operated on by metrorrhagia and/or polyps. Based on this, we recommend the routine use of preoperative biopsy in patients with metrorrhagia and frozen section biopsy in those with polyps.

\section{KEY WORDS: Hysterectomy, uterine pathology, fibroids, endometrial polyps, incidental cancer}

\section{INTRODUCCIÓN}

Dentro de los procedimientos ginecológicos mayores, la histerectomía constituye la cirugía más frecuentemente realizada en los países desarrollados $(1,2)$. En Estados Unidos se estima que se realizan 600.000 histerectomías cada año y que a la edad de 60 años aproximadamente un tercio de las mujeres habrá sido sometida a esta intervención (3). En países con registro adecuado y dependiendo de factores geográficos, factores propios de la paciente o de causa médica, la tasa de histerectomías oscila entre 5,4 por 1000 mujeres en EEUU a 1,8 por 1000 en Noruega (4-7).

Al analizar las causas que motivan la realización de una histerectomía, históricamente, más del $90 \%$ de ellas obedece a una causa benigna, habitualmente leiomioma, adenomiosis y defectos del piso pelviano. Sólo un $10 \%$ de las histerectomías se realizan en contexto de enfermedad maligna ya sea originada en el útero propiamente tal (cuerpo o cuello uterino) o como parte de la etapificación de una neoplasia ginecológica de otro origen $(8,9)$.

Múltiples cuestionamientos han surgido a lo largo del tiempo, particularmente de los administradores o aseguradoras de salud, sobre la correcta indicación de la histerectomía y los costos que de ella derivan (10-16). Resulta difícil establecer indicadores objetivos, excepto los hallazgos en una biopsia, que permitan avalar la correcta indicación de una histerectomía. En nuestro medio, no existen series que hagan referencia al porcentaje de casos de histerectomía con ausencia de hallazgos anátomo-patológicos significativos.

El advenimiento de nuevas tecnologías, tanto en el diagnóstico como en la terapia, han promo- vido el uso de procedimientos menos invasivos y conservadores del cuerpo uterino para condiciones consideradas tradicionalmente causa de histerectomía (17). Dentro de esas condiciones están el leiomioma uterino y la metrorragia disfuncional. Para la primera condición se ha propuesto el uso de la embolización arterial selectiva y para la segunda el uso de métodos ablativos del endometrio como la resectoscopia o la ablación térmica (17). No cabe duda, que es requisito previo a promover su uso, el conocer cuales son los hallazgos anátomo-patológicos en pacientes que fueron histerectomizadas con esos diagnósticos. Resulta importante, el poder establecer la coexistencia de otras enfermedades en la pieza quirúrgica que pudiesen hacer cuestionable la implementación de una terapia conservadora en particular.

Otro tema relevante se refiere el mal manejo de ciertas enfermedades oncológicas, mediante histerectomía exclusiva, producto de un estudio preoperatorio incompleto o la falta de sospecha de cáncer en patologías ginecológicas catalogadas como presuntamente benignas. Para poder conocer cuales condiciones deben ser motivo de mayor sospecha y de una indicación más cauta de histerectomía es necesario conocer los hallazgos anátomo-patológicos en piezas de histerectomía y la ocurrencia de cáncer ginecológico incidental.

De acuerdo a nuestro conocimiento no existen series nacionales que caractericen los hallazgos anátomo-patológicos en pacientes histerectomizadas. En particular, trabajos que analicen la frecuencia relativa de patología uterina benigna y maligna, la coexistencia de patologías y el hallazgo de cáncer incidental originado en el útero.

Los objetivos de este estudio son: 1) caracterizar 
la distribución de la patología uterina originada en el endometrio, miometrio y estroma del cuerpo uterino; 2) determinar la frecuencia en que coexisten patologías, especialmente el hallazgo de cáncer incidental en pacientes operadas por patología presuntamente benigna, y 3) analizar en forma crítica, el porcentaje de casos de histerectomía sin hallazgo de patología ginecológica como un estimado de la correcta indicación quirúrgica.

\section{MATERIAL Y MÉTODO}

Se diseñó un estudio retrospectivo donde se revisaron los registros de biopsias del Departamento de Anatomía Patológica de la Pontificia Universidad Católica de Chile y se reclutaron todos los casos de patología ginecológica operados en el Hospital Clínico en el período comprendido entre el 1 de enero de 1991 y el 31 de diciembre de 2005. Se incluyeron todas las piezas quirúrgicas de útero con o sin extirpación de los anexos. Se incluyeron tanto las histerectomías realizadas por patología uterina como aquellas realizadas por diagnóstico de patología anexial o por un defecto del piso pelviano. Se excluyeron aquellos casos de interconsulta procedentes de otros centros.

La información recopilada fue transferida a una base de datos especialmente diseñada. En ella se incluyeron las siguientes variables: edad, motivo de la cirugía, diagnóstico preoperatorio, tipo de pieza quirúrgica (ej. útero con o sin anexos) e informe anatomopatológico definitivo. Para cada informe se consignaron todos los diagnósticos, tanto del cuerpo uterino como de los anexos u otros órganos extirpados. Los diagnósticos se clasificaron en primer lugar según su localización anatómica y secundariamente según la estirpe histológica. Así, en relación al útero se subdividió la pieza de histerectomía en cuerpo y cuello uterino. A su vez, el cuerpo uterino se subdividió en patología originada en el epitelio (endometrio) o no epitelial (ej. miometrio y estroma). Para la clasificación histológica se estandarizaron los diagnósticos utilizando términos de consenso publicados en textos clásicos y aceptados por la Organización Mundial de la Salud $(18,19)$. Una vez clasificados los diagnósticos se construyeron tablas de frecuencias para la patología uterina estratificadas por edad y/o condición de malignidad. Para ciertos diagnósticos (ej. pólipo) se hizo un análisis adicional en relación al hallazgo de cáncer incidental. El análisis estadístico se realizó mediante los programas JMP 7 (SAS Institute Inc., Cary, NC, EEUU) y Prism 5 for Mac OSX (GraphPad Software, Inc., La Jolla, CA, EEUU). Para el análisis de proporciones entre variables no paramétricas se utilizó el test de Chi cuadrado o de Fisher según correspondiera. Se consideró un valor $p<0,05$ como diferencia significativa.

\section{RESULTADOS}

Durante el período de estudio se realizaron un total de 5.683 histerectomías en nuestro centro. El promedio de edad de las pacientes histerectomizadas fue de 49,6 años con una mediana de 47 años (rango: 18 - 95 años).

La principal indicación de histerectomía fue el leiomioma uterino $(41,4 \%)$. Le siguen en frecuencia la patología anexial $(24,8 \%)$, defecto del piso pelviano $(9,9 \%)$, cáncer ginecológico $(9,5 \%)$, adenomiosis $(5,7 \%)$, metrorragia $(4,4 \%)$, algia pelviana crónica $(1,9 \%)$ y pólipo endometrial $(1 \%)$.

Del total de histerectomías, en el $75,2 \%$ de los casos $(n=4.275)$ se diagnosticó una patología no originada en el epitelio del cuerpo uterino, la mayoría originada en el miometrio (80\%). En algunos casos se encontró más de una patología, totalizando 5.179 diagnósticos. La Tabla I resume los diagnósticos histológicos de las lesiones originadas en los tejidos no epiteliales del cuerpo de útero. El diagnóstico más frecuente fue el tumor benigno originado en el miometrio (leiomioma uterino: 58,7\% de los casos). En un total de 3.334 casos de leiomioma, sólo un $0,14 \%$ de los casos presentó elementos sugerentes de mayor actividad proliferativa (mayor celularidad, presencia de atipías, o mayor índice mitótico). Un 1,3\% de los leiomiomas presentó elementos histopatológicos sugerentes de degeneración, siendo la hialina la más frecuente. Sólo un caso fue catalogado como un tumor del músculo liso de potencial maligno incierto. Dentro de las lesiones originadas en los tejidos no epiteliales del cuerpo uterino, el segundo diagnóstico en frecuencia fue la adenomiosis con un $28,5 \%$. Es importante destacar la coexistencia de adenomiosis y leiomiomas en 909 casos $(27,3 \%$ de los leiomiomas o $56 \%$ de las adenomiosis). Así también, esta entidad coexiste con hiperplasia endometrial (14,7\% de las hiperplasias) y endometriosis (38 casos).

En $36,4 \%$ de los casos de histerectomía se hizo el diagnóstico de patología originada en el epitelio endometrial $(n=2.070)$. Al igual que con la patología de origen no epitelial, hubo casos con más de un diagnóstico de patología endometrial, totalizando 2.279 diagnósticos. La Tabla II muestra los diagnósticos histológicos de las lesiones originadas en el epitelio endometrial. El hallazgo más frecuente fue la atrofia endometrial $(21,7 \%$ de las histerectomías). La atrofia fue del tipo simple en un $79,8 \%$ y de tipo quística en el $20,2 \%$ restante. Le siguen en frecuencia los pólipos endometriales con un 7,4\% (20,4\% de las piezas con patología endometrial), y la hiperplasia endometrial con un $6,6 \%(18,1 \%$ de las piezas con patología endometrial). 
Tabla I

DIAGNÓSTICO HISTOLÓGICO DE LAS LESIONES DE ORIGEN NO EPITELIAL (MIOMETRIO Y ESTROMA) DEL CUERPO UTERINO

\begin{tabular}{|c|c|c|c|}
\hline & $\mathrm{n}$ & $\begin{array}{c}\% \text { total } \\
\text { hallazgos }\end{array}$ & $\begin{array}{c}\% \text { total piezas } \\
\text { quirúrgicas }\end{array}$ \\
\hline \multicolumn{4}{|l|}{ Tumores de músculo liso } \\
\hline Leiomioma & 3282 & 63,4 & 57,8 \\
\hline Leiomioma mitóticamente activo & 2 & 0,04 & 0,04 \\
\hline Leiomioma celular & 5 & 0,1 & 0,1 \\
\hline Leiomioma atípico & 1 & 0,02 & 0,02 \\
\hline Leiomioma mixoideo & 10 & 0,2 & 0,2 \\
\hline Leiomioma con otros elementos & 32 & 0,6 & 0,6 \\
\hline Leiomioma con células hematopoyéticas & 2 & 0,04 & 0,04 \\
\hline \multicolumn{4}{|c|}{ Proliferaciones de músculo liso con patrón inusual de crecimiento } \\
\hline Hipertrofia miometrial & 46 & 0,9 & 0,8 \\
\hline Leiomioma peritoneal o parásito & 11 & 0,2 & 0,2 \\
\hline Leiomiosarcoma & 9 & 0,2 & 0,2 \\
\hline Tumor de músculo liso de potencial maligno incierto & 1 & 0,02 & 0,02 \\
\hline Linfangectasia miometrio & 9 & 0,2 & 0,2 \\
\hline Hiperplasia miometrial & 12 & 0,2 & 0,2 \\
\hline \multicolumn{4}{|l|}{ Tumores del estroma endometrial } \\
\hline Sarcoma del estroma endometrial bajo grado & 2 & 0,04 & 0,04 \\
\hline Sarcoma endometrial & 2 & 0,04 & 0,04 \\
\hline \multicolumn{4}{|l|}{ Tumores mixtos epiteliales- estromales } \\
\hline Adenofibroma & 1 & 0,02 & 0,02 \\
\hline Adenosarcoma & 1 & 0,02 & 0,02 \\
\hline Angiomiolipoma & 1 & 0,02 & 0,02 \\
\hline Tumor mulleriano mixto maligno & 11 & 0,2 & 0,2 \\
\hline \multicolumn{4}{|l|}{ Tumores del mesénquima misceláneos } \\
\hline Esclerosis intersticial del miometrio & 25 & 0,5 & 0,4 \\
\hline Adenomiosis & 1622 & 31,3 & 28,5 \\
\hline Adenomioma & 74 & 1,4 & 1,3 \\
\hline Tumor adenomatoide & 1 & 0,02 & 0,02 \\
\hline Tumor neuroectodérmico primitivo & 1 & 0,02 & 0,02 \\
\hline Otros & 16 & 0,3 & 0,3 \\
\hline
\end{tabular}


Tabla II

DIAGNÓSTICOS HISTOLÓGICOS DE LAS LESIONES ORIGINADAS EN EL EPITELIO ENDOMETRIAL DEL CUERPO UTERINO

\begin{tabular}{llll}
\hline & \% total piezas \\
quirúrgicas
\end{tabular}

Lesiones benignas

Endometritis

- $\quad$ aguda

- crónica inespecífica

- crónica específica

- tuberculosis

Atrofia endometrial

- simple

- quística

Tumores benignos

- póliposimple

- pólipohiperplástico

- $\quad$ adenomioma polipoideo atípico

Hiperplasia endometrial

$$
\begin{array}{ll}
\text { - } & \text { simple } \\
\text { - } & \text { compleja } \\
\text { - } & \text { simple con atipías } \\
\text { - } & \text { compleja con atipías }
\end{array}
$$

Cambios celulares endometriales

- metaplasia escamosa

- metaplasia células claras

Lesiones malignas

- carcinoma endometrial intraepitelial

- adenocarcinoma endometrioide

- $\quad$ adenocarcinoma con diferenciación escamosa

- adenocarcinoma mucinoso

- $\quad$ adenocarcinoma seroso (seroso/papilar)

- adenocarcinoma células claras

- adenocarcinoma de tipo mixto epitelial

- $\quad$ adenocarcinoma indiferenciado

$\begin{array}{ccc}4 & 0,2 & 0,1 \\ 1 & 0,04 & 0,02\end{array}$

0,1

0,02

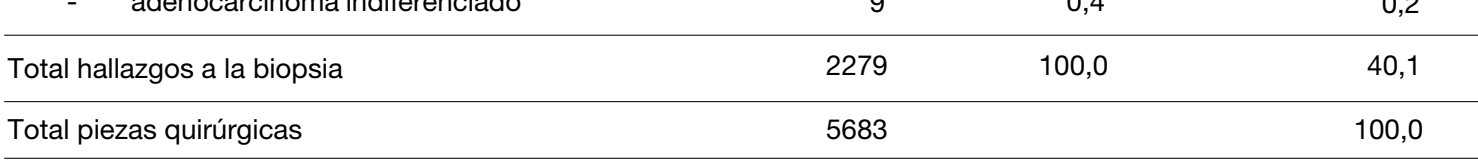


En 905 piezas de histerectomía se demostró la coexistencia de patología originada en el epitelio endometrial y en tejidos no epiteliales del cuerpo uterino (15,9\%).

En sólo 240 histerectomías no se identificó lesión en el examen anatomopatológico (4,2\%). El análisis por diagnóstico preoperatorio demuestra que 75 de estos casos (31,3\%) correspondió a pacientes operadas por un defecto del piso pelviano, donde como parte del tratamiento, se indicó la histerectomía. En 31 casos (12,9\%) el motivo de la cirugía fue una metrorragia disfuncional o la sospecha de hiperplasia endometrial (con o sin biopsia preoperatoria). En 24 casos el motivo fue algia pelviana crónica (10\%). Once casos corresponden a pacientes con patología anexial donde se optó por realizar la histerectomía simultáneamente $(4,6 \%)$. En 16 casos $(6,7 \%)$ el motivo fue el diagnóstico de cáncer (ej. cáncer de endometrio en legrado uterino) sin evidenciarse lesión residual en la pieza de histerectomía. En 73 casos el diagnóstico preoperatorio fue leiomioma $(n=39)$ o adenomiosis $(n=34)$ sin confirmación histológica (representando en conjunto un $30,4 \%)$. Finalmente, 10 casos $(4,2 \%)$ corresponden a histerectomías indicadas por causas misceláneas (ej. paciente con trastorno grave de la coagulación).

Para el análisis de las lesiones malignas la muestra se subdividió en 2 grupos según edad, mujeres menores y mayores de 50 años. En forma arbitraria se consideró 50 años como edad de la menopausia. Ello con el fin de tener una aproximación a las posibles diferencias en la distribución de patología oncológica entre mujeres premenopáusicas y posmenopáusicas.

Respecto de los tumores malignos originados en los tejidos no epiteliales del cuerpo uterino, éstos representan menos del $1 \%$ del total de las histerectomías realizadas en nuestro centro $(0,44 \%$, $\mathrm{n}=25$ ). Los sarcomas uterinos aumentan significativamente su incidencia después de los 50 años, ocurriendo el doble de casos después de esa edad (17 versus 8 casos, $p<0,0007$ ). Dentro de los sarcomas uterinos la variedad histológica más frecuente es el tumor mulleriano mixto (11 casos), todos después de los 50 años. Le siguen en frecuencia el leiomiosarcoma (9 casos). La Tabla III resume los tumores malignos originados en los tejidos no epiteliales del cuerpo uterino.

Los tumores malignos originados en el epitelio endometrial del cuerpo uterino representan el 3\% del total de las piezas de histerectomía $(n=170)$ y el $8,2 \%$ de los úteros con diagnóstico de patología endometrial. El $86 \%$ de los casos de cáncer de endometrio se presenta después de los 50 años. Por tanto, el cáncer de endometrio afecta 6 veces más a mujeres posmenopáusicas (147 vs. 23 pacientes, $\mathrm{p}<0,0001)$. En nuestra serie sólo 7 casos de cáncer de endometrio se presentaron antes de los 40 años lo que representa el $4,1 \%$ del total de casos de cáncer de endometrio (5 casos de carcinoma endometrioide, un carcinoma con diferenciación escamosa y un caso de carcinoma indiferenciado). La variedad histológica más frecuente fue el carcinoma endometrioide $(n=112)$, representando el $77,7 \%$ del total de casos. La coexistencia de cáncer de endometrio con otros cánceres ginecológicos fue excepcional. En esta serie sólo en una paciente se demostró coexistencia con otra neoplasia ginecológica (un caso en que coexistía un tumor de bajo potencial maligno del ovario variedad seroso papilar). En la Tabla IV se resume la distribución de neoplasias malignas originadas en el epitelio endometrial.

Se analizaron aquellos casos operados por patología presuntamente benigna y en los cuales se encontró un cáncer ginecológico incidental. Se incluyeron los casos operados con diagnóstico preoperatorio de leiomioma uterino, adenomiosis, metrorragia disfuncional, pólipo endometrial, prolapso y también aquellos casos de metrorragia con biopsia preoperatoria y diagnóstico de hiperplasia. Del análisis se excluyeron todos aquellos casos con antecedente de cáncer ginecológico y las pacientes con diagnóstico de tumor anexial (por su potencial oncológico). La Tabla $\mathrm{V}$, resume los casos de cáncer ginecológico incidental en pacientes operadas por estas condiciones. En total hubo 35 casos de cáncer incidental representando el $1 \%$ de los casos operados por las condiciones antes mencionadas. De un total de 537 casos de cánceres ginecológicos, incluyendo cánceres originados en el cuerpo (endometrio y sarcomas), cuello uterino, ovario y trompa, los casos de cáncer incidental representan el $6,5 \%$.

Los diagnósticos preoperatorios asociados con mayor frecuencia a cáncer incidental fueron la metrorragia $(5,2 \%)$ y el pólipo endometrial $(3,5 \%)$. Respecto de los casos de cáncer incidental en pacientes con metrorragia, la mayoría correspondieron a cánceres de endometrio (10 de 13 casos). Para los casos de pólipo endometrial, un caso correspondió a un sarcoma uterino y el otro a un cáncer de endometrio (Tabla V). 
Tabla III

DISTRIBUCIÓN DE LAS LESIONES MALIGNAS DE ORIGEN NO EPITELIAL DEL CUERPO UTERINO SEGÚN EDAD DE LA MUJER

\begin{tabular}{lcccc}
\hline & $\begin{array}{c}\leq 50 \text { años } \\
\mathrm{n}(\%)\end{array}$ & $\begin{array}{c}>50 \text { años } \\
\mathrm{n}(\%)\end{array}$ & $\begin{array}{c}\% \text { total } \\
\text { neoplasias }\end{array}$ & $\begin{array}{c}\% \text { total } \\
\text { piezas } \\
\text { (n: 5683) }\end{array}$ \\
\hline $\begin{array}{l}\text { Leiomiosarcoma } \\
\text { Sarcoma del estroma endometrial } \\
\text { de bajo grado }\end{array}$ & $4(50)$ & $5(29,4)$ & 36 & 0,16 \\
Sarcoma endometrial & $2(25)$ & - & 8 & 0,08 \\
Adenosarcoma & $1(12,5)$ & $1(5,9)$ & 8 & 0,08 \\
Tumor mulleriano mixto maligno & $1(12,5)$ & - & 4 & 0,04 \\
Total & - & $11(64,7)$ & 44 & 0,2 \\
& $8(32)$ & $17(68)$ & 100 & 0,44 \\
\hline
\end{tabular}

Tabla IV

DISTRIBUCIÓN DE LAS LESIONES PRECURSORAS Y MALIGNAS ORIGINADAS EN EL EPITELIO ENDOMETRIAL DEL CUERPO UTERINO

\begin{tabular}{ccccc}
\hline & 50 años & $>50$ años & $\%$ total & $\%$ piezas \\
$n(\%)$ & neoplasias & quirúrgicas \\
\hline
\end{tabular}

\section{Lesiones precursoras}

Hiperplasia endometrial

- simple

$208(97,2)$
$159(74,3)$
$5(2,3)$
$36(16,8)$
$8(3,7)$

$167(99,4)$
$122(72,6)$
$6(3,6)$
$29(17,3)$
$10(6,0)$

98,2

6,6

- compleja

- simple con atipias

- compleja con atipias

$4(1,9)$

$1(0,5)$

$1(0,5)$

Carcinoma endometrial intraepitelial

(1)

Lesiones malignas

- adenocarcinoma endometrioide

$18(78,3)$

$94(63,9)$

65,9

2,0

- adenocacinoma endometrioide

con diferenciación escamosa

- adenocarcinoma mucinoso

$3(13,0)$

$$
17(11,7)
$$

11,8

0,4

- adenocarcinoma seroso papilar

- adenocarcinoma células claras

$6(4,1)$

3,5

0,1

- $\quad 14(9,5)$

8,2

0,2

$5(3,4)$

2,9

0,1

- $\quad$ adenocarcinoma de tipo mixto epitelial

$4(2,7)$

2,4

0,1

- adenocarcinoma indiferenciado

$2(8,7)$

$7(4,8)$

5,3

0,2 


\section{Tabla V \\ DISTRIBUCIÓN DEL HALLAZGO INCIDENTAL DE CÁNCER ENTRE PACIENTES OPERADAS POR PATOLOGÍA GINECOLÓGICA PRESUNTAMENTE BENIGNA}

\begin{tabular}{lccccccr}
\hline $\begin{array}{l}\text { Tipo de } \\
\text { cáncer }\end{array}$ & Leiomioma & Prolapso & Adenomiosis & $\begin{array}{c}\text { Diagnóstico preoperatorio } \\
\text { pelviana }\end{array}$ & Metrorragia & Pólipo & $\begin{array}{c}\text { Cáncer } \\
\text { hallazgo } \\
\text { incidental (\%) }\end{array}$ \\
\hline $\begin{array}{l}\text { Endometrio } \\
\text { Sarcoma }\end{array}$ & 6 & - & - & - & 10 & 1 & $17 / 170(10)$ \\
$\begin{array}{l}\text { Cuello } \\
\text { uterino }\end{array}$ & 4 & - & - & - & - & 1 & $5 / 25(20)$ \\
$\begin{array}{l}\text { Ovario } \\
\text { Trompa }\end{array}$ & 1 & - & - & - & 3 & - & $4 / 144(2,8)$ \\
$\begin{array}{l}\text { Cáncer } \\
\text { incidental }\end{array}$ & $15 / 2350$ & $2 / 564$ & $2 / 329$ & $1 / 108$ & $13 / 251$ & $2 / 57$ & $5 / 193(4,7)$ \\
$(\%)$ & $(0,6)$ & $(0,4)$ & $(0,6)$ & $(0,9)$ & $(5,2)$ & $(3,5)$ & 5 \\
\hline
\end{tabular}

El sarcoma uterino fue la neoplasia ginecológica que más frecuentemente se diagnosticó en forma incidental ( $20 \%$ de los casos). De ellos 4 casos se operaron con el diagnóstico de leiomioma uterino y uno como pólipo endometrial. Un 10\% de los cánceres de endometrio fueron diagnosticados incidentalmente, la mayoría en relación a diagnóstico de metrorragia (10 casos) y leiomioma uterino (6 casos). Respecto de otras neoplasias ginecológicas, sólo un $4,7 \%$ de los cánceres de ovario y un $2,8 \%$ de los cánceres de cuello uterino fueron diagnosticados de esta forma.

Respecto de las pacientes operadas con diagnóstico de hiperplasia endometrial $(n=375)$, en $3,7 \%$ de los casos se encontró un cáncer de endometrio. La mayor asociación se estableció con el patrón arquitectural complejo de la hiperplasia independiente de la presencia de atipías (hiperplasia: compleja $20,6 \%$ versus simple $2,3 \%, p<0,0002$ ). La presencia de atipías, independiente del patrón arquitectural, se asoció a un aumento no significativo de los casos de cáncer. Al analizar todos los casos de cáncer de endometrio, en 8,2\% coexistía hiperplasia. La Tabla VI resumen la asociación entre hiperplasia y cáncer de endometrio.

En nuestra serie se registraron 422 casos de histerectomía con presencia de pólipo endometrial, representando $7,4 \%$ del total de histerectomías. Sólo en el $13,5 \%$ de ellos, el pólipo se consignó como indicación de la histerectomía. En 2,4\% de los casos se demostró la presencia de cáncer. En 9 casos se encontró cáncer de endometrio (solo en uno de ellos como hallazgo incidental) y en 1 caso un sarcoma incidental. Al analizar el tipo de pólipo endometrial, un $86,7 \%$ era del tipo simple y el $13,3 \%$ restante de la variedad hiperplásica. Es esta última variedad la que se asocia con mayor frecuencia a cáncer de endometrio (pólipo hiperplásico $3,6 \%$ versus $1,9 \%$ para pólipo simple, $p=N S$ ). Por último, en los 170 casos de cáncer endometrial en un $5,3 \%$ se demostró la presencia de un pólipo endometrial. La Tabla VII resume la asociación entre pólipo y cáncer de endometrio.

Finalmente, el presente trabajo permitió estimar la incidencia de malformaciones müllerianas en pacientes sometidas a histerectomía. En total hubo 9 casos de alteraciones estructurales, representando menos del $0,2 \%$ del total. El defecto de fusión más frecuentemente encontrado fue el útero bicorne unicollis (5 casos), seguido por útero didelfo, bicorne bicollis, arcuato y septado, con un caso cada uno de ellos. 
Tabla VI

HALLAZGO DE CÁNCER DE ENDOMETRIO (170 CASOS) EN PACIENTES OPERADAS POR HIPERPLASIA ENDOMETRIAL

\begin{tabular}{|c|c|c|c|c|c|c|}
\hline Adenocarcinoma & HS & $\mathrm{HC}$ & HSA & $\mathrm{HCA}$ & $\begin{array}{c}\text { HE según } \\
\text { histología cáncer } \\
\mathrm{n}(\%)\end{array}$ & $\begin{array}{l}\text { Total } \\
\text { cáncer }\end{array}$ \\
\hline Endometrioide & 5 & 2 & 1 & 4 & $12(10,7)$ & 112 \\
\hline $\begin{array}{l}\text { Endometrioide con } \\
\text { diferenciación escamosa }\end{array}$ & - & - & - & - & - & 20 \\
\hline Mucinoso & - & - & 1 & - & $1(16,7)$ & 6 \\
\hline Seroso & 1 & - & - & - & $1(7,1)$ & 14 \\
\hline Células claras & - & - & - & - & - & 5 \\
\hline Tipo mixto epitelial & - & - & - & - & - & 4 \\
\hline Indiferenciado & - & - & - & - & - & 9 \\
\hline Total HE y cáncer & 6 & 2 & 2 & 4 & 14 & - \\
\hline Total HE & 281 & 11 & 65 & 18 & - & - \\
\hline$\%$ cáncer del total HE & 2,1 & 18,2 & 3,1 & 22,2 & - & - \\
\hline
\end{tabular}

HE: hiperplasia endometrial. HS: hiperplasia simple. HC: hiperplasia compleja. HSA: hiperplasia simple con atipias. HCA: hiperplasia compleja con atipias

Tabla VII

HALLAZGO DE CÁNCER DE ENDOMETRIO EN PACIENTES OPERADAS POR PÓLIPO ENDOMETRIAL

\begin{tabular}{|c|c|c|c|c|c|}
\hline Adenocarcinoma & $\begin{array}{l}\text { Pólipo } \\
\text { simple }\end{array}$ & $\begin{array}{c}\text { Pólipo } \\
\text { hiperplásico }\end{array}$ & $\begin{array}{l}\text { Total pólipos } \\
\text { asociados a } \\
\text { cáncer }\end{array}$ & $\begin{array}{l}\text { Total } \\
\text { cáncer }\end{array}$ & $\begin{array}{l}\text { Cáncer y } \\
\text { presencia } \\
\text { pólipo (\%) }\end{array}$ \\
\hline Endometrioide & 4 & 2 & 6 & 112 & 5,4 \\
\hline $\begin{array}{l}\text { Endometrioide con } \\
\text { diferenciación escamosa }\end{array}$ & - & - & - & 20 & - \\
\hline Mucinoso & 1 & - & 1 & 6 & 16,7 \\
\hline Seroso & - & - & - & 14 & - \\
\hline Células claras & - & - & - & 5 & - \\
\hline Tipo mixto epitelial & - & - & - & 4 & - \\
\hline Indiferenciado & 2 & - & 2 & 9 & 22,2 \\
\hline Total pólipos y cáncer & 7 & 2 & 9 & 170 & 5,3 \\
\hline
\end{tabular}




\section{DISCUSIÓN}

En la actualidad la histerectomía continúa siendo la cirugía ginecológica más realizada. Al igual que en la mayoría de las series internacionales, la principal indicación para realizar esta intervención es la presencia de leiomiomas sintomáticos (7). En nuestra serie, le sigue en frecuencia la patología anexial en donde muchas veces la histerectomía se realiza por necesidades técnicas (ej. tumor anexial íntimamente adherido al cuerpo uterino) o por coexistencia de patología anexial y uterina (ej. absceso tubo ovárico y miometritis, endometriosis severa). Otras causas frecuentes son los defectos del piso pelviano, los cánceres ginecológicos, la adenomiosis, la metrorragia y el algia pelviana crónica. Al comparar la frecuencia relativa de cada una de estas causas con la reportada por series internacionales observamos que la distribución es similar $(20,21)$. La mayoría de las series coinciden en que un tercio de las histerectomías se realiza por leiomiomas uterinos (representando en nuestra serie poco más del $40 \%$ de los casos) y que otras causas frecuentes son la adenomiosis (con o sin endometriosis coexistente), el sangrado uterino disfuncional, los defectos del piso pelviano, el algia pelviana y la hiperplasia endometrial (22-25). Aunque la distribución es similar, existen algunas diferencias con nuestra serie que pueden explicarse por la no inclusión, en algunas de dichas series, de casos en que la histerectomía se indicó primariamente por patología anexial (coexistiendo patología uterina) y/o aquellos casos con indicación primaria oncológica.

Al momento de decidirse a realizar esta intervención es importante considerar que para la mayoría de los escenarios clínicos las razones que llevan a recomendarla se basan en opiniones de expertos más que en estudios bien diseñados. Siguiendo los principios de la medicina basada en la evidencia, existe respaldo suficiente (tipo IA) que avala su uso como tratamiento de los leiomiomas uterinos, de las lesiones premalignas originas en el útero (hiperplasia endometrial con atipías) y las lesiones malignas de endometrio, cuello uterino, ovario y trompa (1). Para el resto de las condiciones, los elementos más importantes al momento de definir la necesidad y el momento de su indicación son los síntomas que refiere la paciente y el juicio clínico del médico tratante. Por tanto cada vez que se decide por ella debe tomarse en cuenta previamente cuáles son los riesgos y beneficios inherentes al procedimiento, cuáles son las preferencias de la paciente (particularmente si hay deseo de preservar el potencial reproductivo o simplemente de preservar la indemnidad de sus órganos genitales) y si hay otras alternativas menos radicales por ofre- cer o que hayan fallado previamente $(17,26)$.

Al analizar cuáles son las mujeres que son sometidas a este procedimiento, observamos que se trata de mujeres habitualmente mayores de 40 años. Esto es coincidente con lo reportado internacionalmente donde el grupo que concentra el mayor número de procedimientos está entre los 40 y 49 años $(7,27)$. Esta distribución etárea se explica probablemente por la presencia de patología sintomática, considerada hormonalmente dependiente (ej., leiomiomas e hiperplasia), en mujeres que han completado su paridad y que prefieren un tratamiento definitivo a su enfermedad.

Coincidente con la frecuencia en el diagnóstico preoperatorio, la etiología más frecuentemente diagnosticada fue el leiomioma uterino, representando el $58 \%$ de los diagnósticos. La diferencia entre frecuencias, comparando el diagnóstico preoperatorio y la patología definitiva, se explica por la coexistencia de leiomiomas uterinos en pacientes operadas con otro diagnóstico (ej. tumor anexial). Diferencias similares se describen en series internacionales en donde los leiomiomas uterinos se manifiestan en forma sintomática en hasta $25 \%$ de las mujeres en edad reproductiva y son diagnosticados en el 70 a $80 \%$ de las piezas de histerectomía (28). Los dos hallazgos que siguen en frecuencia al leiomioma uterino son la adenomiosis y atrofia endometrial, presentes en un $28 \%$ y $21 \%$ respectivamente. $\mathrm{Al}$ analizar el conjunto de causas más frecuentemente encontradas, éstas son coincidentes con las reportadas por series internacionales (29).

Una patología originada en los tejidos no epiteliales para la cual no existe claridad sobre su real incidencia y origen etiológico es la adenomiosis. Esta entidad se define como la presencia de glándulas y estroma endometrial en el espesor del miometrio (30). Un símil al endometrio ectópico presente en la endometriosis ovárica pero localizada en el cuerpo uterino. Dada su localización, para estimar su real incidencia es requisito realizar examen histológico del cuerpo uterino que confirme su presencia. Estimados de series internacionales establecen su incidencia entre un 9 y 62\% (21, 31-33). En nuestra serie, poco menos del $30 \%$ de las piezas de histerectomía presentaron adenomiosis. Al igual que la endometriosis, se considera a esta entidad como una patología hormonodependiente en donde la acción de los estrógenos y progesterona afectan la actividad y sintomatología generada por la enfermedad. Dentro de las opciones terapéuticas para mujeres afectadas por esta condición está la histerectomía.

Frente al surgimiento de métodos menos invasivos y más conservadores para el manejo de ciertas patologías originadas en el cuerpo uterino 
(ej. embolización arterial selectiva de leiomiomas, resectoscopia en casos de leiomioma submucoso o pólipos) es importante destacar la frecuente coexistencia de ciertas patologías que eventualmente pudiesen afectar el éxito de dichos métodos (17). Esto es particularmente importante cuando se trata de mujeres en quienes el deseo de paridad no constituye un problema. Tal es el caso de la adenomiosis (30). En nuestra serie observamos la coexistencia de adenomiosis y leiomioma uterino en poco menos del $30 \%$ de los casos. En tal escenario, limitar el tratamiento a una miomectomía pudiese eventualmente significar el fracaso terapéutico. Tal fracaso no sólo ocurriría por la recurrencia de un leiomioma a posteriori sino también por la persistencia de síntomas, como es la dismenorrea en el caso de la adenomiosis. También observamos en nuestra serie la coexistencia de patología endometrial y del miometrio (ej. $15 \%$ de los casos de hiperplasia endometrial presentan adenomiosis) en donde el tratamiento de una condición pudiese dejar sin tratamiento a la otra. Por ejemplo realizar una resectoscopia por un pólipo sintomático o un legrado uterino por hiperplasia endometrial cuando coexiste adenomiosis. Nuestros resultados son coincidentes con series extranjeras reportando que $37 \%$ de los úteros con leiomiomas presentan adenomiosis y que úteros con hiperplasia tienen 3 veces mayor riesgo de tener adenomiosis $(33,34)$. Por tanto, al momento de optar por una modalidad terapéutica conservadora frente a leiomiomas o hiperplasia endometrial, tanto el médico como la paciente deben determinar si esa opción es la mejor para la paciente particularmente si hay sospecha o presencia de adenomiosis (30).

Al analizar la patología uterina maligna originada en el mesénquima observamos que la distribución según histologías en nuestra serie es diferente a la reportada por otras donde el leiomiosarcoma es la variedad más frecuente (35). Observamos que el tumor más frecuentemente encontrado es el tumor mulleriano mixto maligno (TMMM), en su mayoría, casos de carcinosarcoma (11 casos, representando poco menos del $45 \%$ del total de sarcomas). Le sigue en frecuencia el leiomiosarcoma con 9 casos (representando poco menos del $40 \%$ del total de sarcomas). Es importante señalar que todos los casos de TMMM se presentaron en mujeres mayores de 50 años, esto es en la postmenopausia. En cambio los leiomiosarcomas se distribuyeron equitativamente entre mujeres menores y mayores de esa edad. Otras variedades como el sarcoma originado en el estroma endometrial también se presentaron mayoritariamente en mujeres menores de 50 años. En globo, encontramos 25 casos de sarcoma en esta serie, lo que representa $0,4 \%$ del total de piezas de histerectomía. Si se trata de establecer el riesgo de encontrar un sarcoma uterino en el contexto de leiomioma uterino, este es menor del $1 \%(0,3 \%$ del total de leiomiomas, incluidos solo los leiomiosarcomas) (ver Tabla III). Estos resultados son similares a lo reportado por otras series (3639).

Dentro de las neoplasias ginecológicas, el adenocarcinoma de endometrio constituye el cáncer ginecológico más frecuente en los países desarrollados. En nuestra serie, el cáncer de endometrio representa el 3\% del total de piezas de histerectomía. La variedad histológica más frecuente es el adenocarcinoma endometrioide $(77,7 \%)$ siendo seguido por el adenocarcinoma seroso/papilar $(8,2 \%)$ y el adenocarcinoma indiferenciado $(5,3 \%)$ (ver Tabla IV). La mayoría de los cánceres de endometrio se presentaron en mujeres mayores de 50 años (83,9\% de los casos). La incidencia de cáncer de endometrio en pacientes menores de 40 años fue de un $4,1 \%$, similar al $5 \%$ descrito por series extranjeras (40).

Dos lesiones consideradas hormonodependientes, el pólipo endometrial y la hiperplasia endometrial, se presentaron en el $7,4 \%$ y $6,6 \%$ de las piezas de histerectomías, respectivamente (41-43). Para ambas entidades se ha descrito una mayor asociación con cáncer de endometrio (44). Pero es a la hiperplasia endometrial a la que se considera una lesión precursora. En la historia natural del cáncer de endometrio se ha propuesto un continuo desde lesiones de poca complejidad arquitectural (la hiperplasia simple con o sin atipías), pasando por lesiones de mayor complejidad (la hiperplasia compleja con o sin atipías) hasta el cáncer de endometrio propiamente tal (45). Tradicionalmente se considera que a mayor complejidad arquitectural de la hiperplasia, en presencia de atipías, la coexistencia de un cáncer de endometrio alcanza el 40 a $50 \%$ de las pacientes (46-48). En nuestra serie observamos que es la complejidad arquitectural de la hiperplasia la que se asocia con mayor frecuencia a cáncer de endometrio (asociación que alcanza un $22,2 \%$ en presencia de una hiperplasia endometrial compleja con atipías) (ver Tabla VI). Tanto para la hiperplasia simple como para la compleja, la presencia de atipías aumenta de manera no significativa la asociación con cáncer de endometrio. Del total de cánceres de endometrio registrados, en $8,2 \%$ coexistía la hiperplasia en el endometrio circundante. En relación al pólipo endometrial y su asociación con cáncer de endometrio, se desconoce realmente si el pólipo es una lesión precursora de este cáncer. Se ha propuesto como un factor de riesgo, por encontrarse adyacente a lesiones precursoras como la hiperplasia (49). Un estudio italiano que evaluó 
la presencia de hiperplasia y cáncer en pacientes sometidas a polipectomía por vía histeroscópica describió que un $70 \%$ de los pólipos son benignos, un $28,8 \%$ presenta hiperplasia y en un $0,7 \%$ se encuentra cáncer (49). En series internacionales se describe la degeneración maligna primaria de un pólipo y su incidencia oscila entre un 0,5 y $4,8 \%$ de los casos (50-54). En nuestra serie, en un 5,3\% de los casos de cáncer de endometrio se encontró un pólipo endometrial. Un 13,3\% de los pólipos se definió histológicamente como de tipo hiperplásico, siendo dicha variedad la más frecuentemente asociada a cáncer de endometrio (3,6\% versus $1,9 \%$ comparado con pólipo simple). El porcentaje de asociación encontrado es similar a lo descrito por otras series. Es importante señalar que estudios recientes muestran que la posibilidad de encontrar cáncer es similar entre pacientes portadoras de pólipo endometrial sintomáticas o no $(52,55)$. Tradicionalmente en nuestro grupo hemos considerado la extirpación de pólipos sólo en presencia de síntomas (metrorragia). La asociación encontrada, nos ha hecho replantear tal conducta y ha motivado un estudio, actualmente en curso, para determinar cual es la mejor opción de manejo para pacientes con pólipo endometrial asintomático y sin deseos de paridad futura. Basado en nuestros resultados, por el momento, no parece equivocado ofrecer una histerectomía a una paciente portadora de un pólipo asintomático (hecho particularmente importante en mujeres tratadas por cáncer de mama y con el antecedente de uso de tamoxifeno) (56). En aquellas pacientes que desean preservar la fertilidad, es recomendable considerar la visión directa de la lesión, mediante histeroscopia, a fin de resecar la lesión y obtener una muestra del endometrio circundante o de cualquier área sospechosa.

Un elemento central en el manejo quirúrgico correcto de las neoplasias ginecológicas es la realización de la intervención apropiada y oportuna para el caso particular según la etapa de presentación al momento del diagnóstico. No cabe duda que realizar una histerectomía simple en un cáncer de cuello uterino en etapa precoz puede resultar en un tratamiento insuficiente que afecte la sobrevida y que por ende requiera de terapias complementarias, en muchas ocasiones menos efectivas y con mayor morbilidad asociada $(57,58)$. Por tanto, resulta esencial, previo a indicar una histerectomía, el realizar el mayor estudio que garantice el menor riesgo de encontrar un cáncer incidental en una paciente operada por una patología presuntamente benigna. Poca información existe que establezca cual es la incidencia de cáncer incidental en este escenario $(59,60)$. El hallazgo de lesiones malignas en úteros operados por condición supuestamente benigna depende del diagnóstico preoperatorio. Tal como lo muestra la Tabla $\mathrm{V}$, los dos diagnósticos con mayor riesgo de cáncer incidental son aquellas pacientes operadas por metrorragia y pólipo endometrial en donde se encontró cáncer en un 5,2\% y $3,5 \%$ respectivamente. Por el contrario si la causa de la cirugía es un prolapso uterino, la ocurrencia se cáncer incidental se reduce al $1 \%$, principalmente en el grupo de pacientes sobre 65 años. Para este grupo los principales hallazgos encontrados son el leiomioma (44\%), la adenomiosis $(27,2 \%)$ y el pólipo endometrial (23,9\%). Tradicionalmente se ha postulado que en úteros macroscópicamente sanos no sería necesario el estudio histológico contemporáneo, ya que tal estudio no modifica el manejo posterior, dada la baja frecuencia de cáncer encontrado en dichas piezas $(35,61)$. Sin embargo, creemos conveniente recomendar el abrir el útero, visualizar la cavidad y considerar la biopsia intraoperatoria frente a cualquier lesión sospechosa, particularmente si el diagnóstico preoperatorio es metrorragia o pólipo. Es importante recalcar que frente a la metrorragia siempre debe realizarse estudio biópsico preoperatorio, particularmente en mujeres posmenopáusicas donde la metrorragia se ha asociado con un 4,4\% de probabilidad de tener un cáncer de endometrio (62) (similar al 4\% encontrado en nuestra serie).

Respecto de cuáles cánceres ginecológicos se diagnostican más frecuentemente de manera incidental, en nuestra serie, los sarcomas uterinos son un hallazgo en un $20 \%$ de los casos, la mayoría siendo operados con el diagnóstico de leiomioma uterino ( $80 \%$ de los casos). Le siguen en frecuencia, el cáncer de endometrio (10\% de los casos), la mayoría operados con diagnóstico de metrorragia ( $91 \%$ de los casos). A fin de disminuir tal porcentaje de cáncer incidental en ambas patologías es necesario definir criterios clínicos de sospecha. Debe sospecharse un sarcoma frente a un leiomioma de crecimiento rápido particularmente en mujeres posmenopáusicas refiriendo dolor y/o compromiso del estado general. A fin de evitar un cáncer de endometrio, siempre debe hacerse biopsia de endometrio previo a la cirugía por metrorragia.

De acuerdo a nuestro conocimiento no existe información nacional sobre la incidencia de malformaciones uterinas. Series internacionales establecen que entre un 0,1 a $2 \%$ de las mujeres es portadora de alguna alteración del desarrollo de los conductos paramesonéfricos comprendiendo una amplia gama de fenotipos (63). Según la clasificación de la Sociedad Americana de Fertilidad el defecto más frecuente es el útero septado (64). Sin 
embargo, creemos que existe un sesgo en tal información dado que se trata de una población seleccionada, habitualmente mujeres con problemas de fertilidad $(63,65,66)$. En nuestra serie encontramos 9 casos de malformación uterina, todos hallazgos de la cirugía, representando alrededor del 0,2\% del total de piezas de histerectomía. En esta serie la malformación más frecuentemente encontrada fue el útero bicorne.

Definir cuál debe considerarse una tasa adecuada de histerectomías para una determinada población es materia de debate. Los avances en el diagnóstico por imágenes y el desarrollo de nuevas terapias que permiten un manejo conservador para patologías consideradas tradicionalmente indicación de histerectomía (ej. leiomioma uterino y sangrado uterino disfuncional) hacen más difícil establecer cual debe ser dicha tasa. Más que definir una tasa (variable entre centros con distinta tecnología y poblaciones con distintas patologías) es preferible conocer el porcentaje de casos en que la indicación es correcta. Frente a la subjetividad de los síntomas que motivan la cirugía y la disparidad de criterios entre clínicos para indicar una histerectomía (ej. leiomioma uterino) el único estimador confiable de una indicación correcta es la confirmación de algún hallazgo patológico en la pieza quirúrgica (26). Al respecto no es infrecuente que en pacientes operadas por prolapso o algia pelviana crónica no se encuentre lesión en el examen anatomopatológico (1). En nuestra serie sólo en 240 casos no se demostró patología alguna en la pieza, representando alrededor del $4 \%$ del total de histerectomías. La mayoría de los casos correspondió a pacientes con defectos del piso pelviano (31\% de los casos), escenario donde el clínico consideró necesaria la histerectomía para un tratamiento correcto del defecto. Nuestros resultados son adecuados comparados con otras series internacionales (67). De un análisis crítico de los casos, creemos que es posible reducir al menos en un tercio el porcentaje encontrado, independiente de los síntomas que hayan motivado la cirugía, solo si se mejora el enfrentamiento diagnóstico y terapéutico de casos de leiomioma o adenomiosis sin correlación histológica (68). A modo de ejemplo, en dichos casos, si el motivo hubiese sido la hipermenorrea, una alternativa habría sido la ablación endometrial previo estudio biópsico del endometrio (69).

\section{CONCLUSIÓN}

Esta serie demuestra que la distribución de la patología uterina en pacientes sometidas a histerectomía en un centro universitario sigue un patrón similar al descrito por series internacionales. Para la mayoría de los casos la indicación de histerectomía es correcta ( $96 \%$ de los casos). Creemos que en ello contribuye el ambiente de discusión que se genera en las reuniones de comité, en las cuales cada caso es sometido al análisis crítico por parte de un grupo de médicos expertos, capacitados en las distintas modalidades de tratamiento (conservadores o no). Este trabajo también demuestra que, en globo, el hallazgo de cáncer incidental es menor al 1\%. Dicha cifra aumenta significativamente cuando la causa de la cirugía es la metrorragia o el pólipo endometrial. Ello nos lleva a recomendar el uso rutinario del estudio biópsico preoperatorio en pacientes con metrorragia y de la biopsia contemporánea en casos de pólipo endometrial.

\section{BIBLIOGRAFÍA}

1. Lefebvre G, Allaire C, Jeffrey J, et al. SOGC clinical guidelines. Hysterectomy. J Obstet Gynaecol Can 2002;24(1):37-61.

2. Kozak LJ, Owings MF, Hall MJ. National Hospital Discharge Survey: 2002 annual summary with detailed diagnosis and procedure data. Vital Health Stat 13. 2005(158):1-199.

3. Weaver F, Hynes D, Goldberg JM, Khuri S, Daley J, Henderson W. Hysterectomy in Veterans Affairs Medical Centers. Obstet Gynecol 2001;97(6):880-4.

4. Farquhar CM, Steiner CA. Hysterectomy rates in the United States 1990-1997. Obstet Gynecol 2002;99(2):229-34.

5. McPherson K, Wennberg JE, Hovind OB, Clifford $\mathrm{P}$. Small-area variations in the use of common surgical procedures: an international comparison of New England, England, and Norway. N Engl J Med 1982;307(21):13104.

6. Spilsbury K, Semmens JB, Hammond I, Bolck A. Persistent high rates of hysterectomy in Western Australia: a population-based study of 83000 procedures over 23 years. BJOG 2006;113(7):804-9.

7. Jacobson GF, Shaber RE, Armstrong MA, Hung YY. Hysterectomy rates for benign indications. Obstet Gynecol 2006;107(6):1278-83.

8. Edozien LC. Hysterectomy for benign conditions. BMJ 2005;330(7506):1457-8.

9. Garry R. Health economics of hysterectomy. Best Pract Res Clin Obstet Gynaecol 2005;19(3):451-65.

10. Goldberg J, Bussard A, McNeil J, Diamond J. Cost and reimbursement for three fibroid treatments: abdominal hysterectomy, abdominal myomectomy, and uterine fibroid embolization. Cardiovasc Intervent Radiol 2007;30(1):54-8.

11. Kaunitz AM, Stern L, Doyle J, Etschmaie MR. Use of the levonorgestrel-IUD in the treatment of menorrhagia: improving patient outcomes while reducing the need for surgical management. Manag Care Interface 2007;20(3):47-50.

12. Dembek CJ, Pelletier EM, Isaacson KB, Spies JB. Payer costs in patients undergoing uterine artery embolization, 
hysterectomy, or myomectomy for treatment of uterine fibroids. J Vasc Interv Radiol 2007;18(10):1207-13.

13. Lee DW, Gibson TB, Carls GS, Ozminkowski RJ, Wang S, Stewart EA. Uterine fibroid treatment patterns in a population of insured women. Fertil Steril 2008. doi:10.1016/j.fertnstert.2007.12.004

14. Subak LL, Waetjen LE, van den Eeden S, Thom DH, Vittinghoff $E$, Brown JS. Cost of pelvic organ prolapse surgery in the United States. Obstet Gynecol 2001;98(4):646-51.

15. Mawson AR. The place of hysterectomy in the management of benign uterine disease. HMO Pract 1996;10(2):69-74.

16. London R, Holzman M, Rubin D, Moffitt B. Payer cost savings with endometrial ablation therapy. Am J Manag Care 1999;5(7):889-97.

17. Scialli AR. Alternatives to hysterectomy for benign conditions. Int J Fertil Womens Med 1998;43(4):18691.

18. Tavassoli FA, Devilee P, eds. World Health Organization Classification of tumours. Pathology and genetics od tumours of the breast and female genital organs. Lyon: IARC Press; 2003.

19. Kurman RJ, ed. Blaustein's pathology of the female genital tract. Fifth edition ed. New York: Springer; 2002.

20. MacKenzie IZ, Naish C, Rees M, Manek S. 1170 consecutive hysterectomies: indications and pathology. J Br Menopause Soc 2004;10(3):108-12.

21. Vavilis D, Agorastos T, Tzafetas J, et al. Adenomyosis at hysterectomy: prevalence and relationship to operative findings and reproductive and menstrual factors. Clin Exp Obstet Gynecol 1997;24(1):36-8.

22. Debodinance $P$. Hysterectomy for benign lesions in the north of France: epidemiology and postoperative events. J Gynecol Obstet Biol Reprod (Paris) 2001;30(2):151-9.

23. Baggish MS. Total and subtotal abdominal hysterectomy. Best Pract Res Clin Obstet Gynaecol 2005;19(3):333-56.

24. Carlson KJ, Nichols DH, Schiff I. Indications for hysterectomy. N Engl J Med 25 1993;328(12):856-60.

25. Sait K, Alkhattabi M, Boker A, Alhashemi J. Hysterectomy for benign conditions in a university hospital in Saudi Arabia. Ann Saudi Med 2008;28(4):282-6.

26. Broder MS, Kanouse DE, Mittman BS, Bernstein SJ. The appropriateness of recommendations for hysterectomy. Obstet Gynecol 2000;95(2):199-205.

27. Ong S, Codd MB, Coughlan M, O'Herlihy C. Prevalence of hysterectomy in Ireland. Int J Gynaecol Obstet 2000;69(3):243-7.

28. Cramer SF, Patel A. The frequency of uterine leiomyomas. Am J Clin Pathol 1990;94(4):435-8.

29. Sobande AA, Eskandar M, Archibong EI, Damole IO. Elective hysterectomy: a clinicopathological review from Abha catchment area of Saudi Arabia. West Afr J Med 2005;24(1):31-5.

30. Matalliotakis IM, Kourtis AI, Panidis DK. Adenomyosis. Obstet Gynecol Clin North Am 2003;30(1):63-82, viii.

31. Yeniel O, Cirpan T, Ulukus M, et al. Adenomyosis: prevalence, risk factors, symptoms and clinical findings. Clin Exp Obstet Gynecol 2007;34(3):163-7.
32. Goswami A, Khemani M, Logani KB, Anand R. Adenomyosis: diagnosis by hysteroscopic endomyometrial biopsy, correlation of incidence and severity with menorrhagia. J Obstet Gynaecol Res 1998;24(4):2814.

33. Bergholt T, Eriksen L, Berendt N, Jacobsen M, Her$\mathrm{tz} \mathrm{JB}$. Prevalence and risk factors of adenomyosis at hysterectomy. Hum Reprod 2001;16(11):2418-21.

34. Yin $\mathrm{H}$, Mittal $\mathrm{K}$. Incidental findings in uterine prolapse specimen: frequency and implications. Int J Gynecol Pathol 2004;23(1):26-8.

35. Acharya S, Hensley ML, Montag AC, Fleming GF. Rare uterine cancers. Lancet Oncol 2005;6(12):96171.

36. Parker WH, Fu YS, Berek JS. Uterine sarcoma in patients operated on for presumed leiomyoma and rapidly growing leiomyoma. Obstet Gynecol 1994;83(3):4148.

37. Leibsohn S, d'Ablaing G, Mishell DR, Jr., Schlaerth $\mathrm{JB}$. Leiomyosarcoma in a series of hysterectomies performed for presumed uterine leiomyomas. Am J Obstet Gynecol 1990;162(4):968-974.

38. Quade BJ, Wang TY, Sornberger K, Dal Cin P, Mutter GL, Morton CC. Molecular pathogenesis of uterine smooth muscle tumors from transcriptional profiling. Genes Chromosomes Cancer 2004;40(2):97-108.

39. Olah KS, Gee H, Blunt S, Dunn JA, Kelly K, Chan KK. Retrospective analysis of 318 cases of uterine sarcoma. Eur J Cancer 1991;27(9):1095-9.

40. Pellerin GP, Finan MA. Endometrial cancer in women 45 years of age or younger: a clinicopathological analysis. Am J Obstet Gynecol 2005;193(5):1640-4.

41. Taylor LJ, Jackson TL, Reid JG, Duffy SR. The differential expression of oestrogen receptors, progesterone receptors, $\mathrm{Bcl}-2$ and $\mathrm{Ki} 67$ in endometrial polyps. BJOG 2003;110(9):794-8.

42. Oguz S, Sargin A, Kelekci S, Aytan H, Tapisiz OL, Mollamahmutoglu $\mathrm{L}$. The role of hormone replacement therapy in endometrial polyp formation. Maturitas 2005;50(3):231-6.

43. Deligdisch L. Hormonal pathology of the endometrium. Mod Pathol 2000;13(3):285-94.

44. Mittal K, Da Costa D. Endometrial hyperplasia and carcinoma in endometrial polyps: clinicopathologic and follow-up findings. Int J Gynecol Pathol 2008;27(1):458.

45. Kurman RJ, Kaminski PF, Norris HJ. The behavior of endometrial hyperplasia. A long-term study of "untreated" hyperplasia in 170 patients. Cancer 15 1985;56(2):40312.

46. Trimble CL, Kauderer J, Zaino R, et al. Concurrent endometrial carcinoma in women with a biopsy diagnosis of atypical endometrial hyperplasia: a Gynecologic Oncology Group study. Cancer 2006;106(4):812-9.

47. Shutter J, Wright TC, Jr. Prevalence of underlying adenocarcinoma in women with atypical endometrial hyperplasia. Int J Gynecol Pathol 2005;24(4):313-8.

48. Merisio C, Berretta R, De loris A, et al. Endometrial cancer in patients with preoperative diagnosis of atypical endometrial hyperplasia. Eur J Obstet Gynecol Reprod Biol 2005;122(1):107-11.

49. Savelli L, De laco P, Santini D, et al. Histopathologic features and risk factors for benignity, hyperplasia, 
and cancer in endometrial polyps. Am J Obstet Gynecol 2003;188(4):927-31.

50. Goldstein SR, Monteagudo A, Popiolek D, Mayberry $\mathrm{P}$, Timor-Tritsch I. Evaluation of endometrial polyps. Am J Obstet Gynecol 2002;186(4):669-74.

51. Wolfe SA, Mackles A. Malignant lesions arising from bening endometrial polyps. Obstet Gynecol 1962;20:54250.

52. Papadia A, Gerbaldo D, Fulcheri E, et al. The risk of premalignant and malignant pathology in endometrial polyps: should every polyp be resected? Minerva Ginecol 2007;59(2):117-24.

53. Shushan A, Revel A, Rojansky N. How often are endometrial polyps malignant? Gynecol Obstet Invest 2004;58(4):212-5.

54. Ben-Arie A, Goldchmit C, Laviv Y, et al. The malignant potential of endometrial polyps. Eur J Obstet Gynecol Reprod Biol 2004;115(2):206-10.

55. Lieng M, Qvigstad E, Sandvik L, Jorgensen H, Langebrekke $\mathrm{A}$, Istre $\mathrm{O}$. Hysteroscopic resection of symptomatic and asymptomatic endometrial polyps. J Minim Invasive Gynecol 2007;14(2):189-94

56. Varras M, Polyzos D, Akrivis C. Effects of tamoxifen on the human female genital tract: review of the literature. Eur J Gynaecol Oncol 2003;24(3-4):258-68.

57. Choi DH, Huh SJ, Nam KH. Radiation therapy results for patients undergoing inappropriate surgery in the presence of invasive cervical carcinoma. Gynecol Oncol 1997;65(3):506-11.

58. Saibishkumar EP, Patel FD, Ghoshal S, Kumar V, Karunanidhi G, Sharma SC. Results of salvage radiotherapy after inadequate surgery in invasive cervical carcinoma patients: a retrospective analysis. Int J Radiat Oncol Biol Phys 2005;63(3):828-33.

59. Orr JW, Jr., Ball GC, Soong SJ, Hatch KD, Partridge $E E$, Austin JM. Surgical treatment of women found to have invasive cervix cancer at the time of total hysterectomy. Obstet Gynecol 1986;68(3):353-6.
60. Chapman JA, Mannel RS, DiSaia PJ, Walker JL, Berman ML. Surgical treatment of unexpected invasive cervical cancer found at total hysterectomy. Obstet Gynecol 1992;80(6):931-4.

61. Salmon HA, Smith JH, Balsitis M. Is microscopic assessment of macroscopically normal hysterectomy specimens necessary? J Clin Pathol 2002;55(1):67-8.

62. Bachmann LM, ter Riet G, Clark TJ, Gupta JK, Khan KS. Probability analysis for diagnosis of endometrial hyperplasia and cancer in postmenopausal bleeding: an approach for a rational diagnostic workup. Acta Obstet Gynecol Scand 2003;82(6):564-9.

63. Nahum GG. Uterine anomalies. How common are they, and what is their distribution among subtypes? J Reprod Med 1998;43(10):877-87.

64. The American Fertility Society classifications of adnexal adhesions, distal tubal occlusion, tubal occlusion secondary to tubal ligation, tubal pregnancies, mullerian anomalies and intrauterine adhesions. Fertil Steril 1988;49(6):944-55.

65. Liu YH, Jain S, Lee CL, Soong YK. Incidence of mullerian defects in fertile and infertile women. $\mathrm{J} \mathrm{Am} \mathrm{Assoc}$ Gynecol Laparosc 2000;7(3):435-6.

66. Shulman LP. Mullerian anomalies. Clin Obstet Gynecol 2008;51(2):214-22.

67. Chao YM, Tseng TC, Su CH, Chien LY. Appropriateness of hysterectomy in Taiwan. J Formos Med Assoc 2005;104(2):107-12.

68. Ascher SM, Jha RC, Reinhold C. Benign myometrial conditions: leiomyomas and adenomyosis. Top Magn Reson Imaging 2003;14(4):281-304.

69. Owusu-Ansah R, Gatongi D, Chien PF. Health technology assessment of surgical therapies for benign gynaecological disease. Best Pract Res Clin Obstet Gynaecol 2006;20(6):841-79. 